\title{
Fuzzy Timed Agent Based Petri Nets for Modeling Cooperative Multi-Robot Systems
}

\author{
Xingli HUANG, Hua XU, Peifa JIA \\ State Key Laboratory on Intelligent Technology and Systems, Tsinghua National Laboratory for \\ Information Science and Technology, Department of Computer Science and Technology, \\ Tsinghua University, Beijing, China \\ E-mail:hx1712@sina.com,xuhua@tsinghua.edu.cn \\ Received September 1, 2009; revised October 11, 2009; accepted November 20, 2009
}

\begin{abstract}
A cooperative multi-robot system (CMRS) modeling method called fuzzy timed agent based Petri nets (FTAPN) is proposed in this paper, which has been extended from fuzzy timed object-oriented Petri net (FTOPN). The proposed FTAPN can be used to model and illustrate both the structural and dynamic aspects of CMRS, which is a typical multi-agent system (MAS). At the same time, supervised learning is supported in FTAPN. As a special type of high-level object, agent is introduced into FTAPN, which is used as a common modeling object in its model. The proposed FTAPN can not only be used to model CMRS and represent system aging effect, but also be refined into the object-oriented implementation easily. At the same time, it can also be regarded as a conceptual and practical artificial intelligence (AI) tool for multi-agent systems (MAS) into the mainstream practice of the software development.
\end{abstract}

Keywords: Petri nets, Multi Cooperative Robot Systems, Multi-Agent Systems

\section{Introduction}

As a kind of typical manufacturing equipment, cooperative multi-robot systems (CMRS) have been widely used in current industries [1]. The key solution for one CMRS is to realize the cooperation, which is different from generic control systems. So, currently, the CMRS modeling, analysis and refinement always meet with difficulties related to the cooperation problem. As one of the typical multi-agent systems (MAS) in distributed artificial intelligence [2], CMRS can be regarded as one MAS. In order to model MAS, some attempts to use object-oriented methodology have been tried and some typical agent objects have been proposed, such as active object, etc [3]. However, agent based object models still can not depict the whole structure and dynamic aspects of MAS, such as cooperation, learning, temporal constraints, etc [2].

On one hand, as one of the most proper and promised realization technologies, object-oriented concurrent programming (OOCP) methodology is always used to realize MAS [3]. In OOCP realization, one special object called active object is proposed [3], which can be used to model generic agent architectures and behaviors with $\mathrm{OO}$ methodology. Although OOCP can solve MAS realization problem favorably, the modeling problem mentioned above still exists.

On the other hand, as a kind of powerful formal description and analysis method for dynamic systems [4], Petri net $(\mathrm{PN})$ has become a bridge between practical application and model theories [4]. Basic Petri nets lack temporal knowledge description, so they have failed to describe the temporal constraints in time critical or time dependent systems. Then in the improved models of Petri nets such as Timed (or Time) Petri nets (TPN) [5,6] etc al, temporal knowledge has been introduced, which has increased not only the modeling power but also the model complexity [7]. On the other hand, when Petri nets are used to analyze and model practical systems in different fields, models may be too complex to be analyzed. These years, object-oriented concepts have been introduced into Petri nets such as object Petri nets (OPN) [8], VDM++ [9], Object-Z [10], etc al. are suggested. Among the studies, the research on OPN has been focused on the extending Petri net formalism to OPN such as HOONet [11], OBJSA [12], COOPN/2 [13] and LOOPN++ [14], which are suggested on the base of colored Petri Net (CPN) [15]. Object-oriented Petri net (OPN) can model various systems hierarchically and the models can be analyzed even if they have not been completed. So the complexity of OPN models can be simplified at the be- 
ginning of modeling stage according to the analysis requirements. Although the results of such studies have shown promise, these nets do not fully support time critical (time dependent) system modeling and analysis, which may be complex, midsize or even small. When time critical systems with any sizes are modeled, it requires formal modeling and analysis method supporting temporal description and object-oriented concepts. Then for providing the ability of modeling time critical complex systems, timed hierarchical object oriented Petri net (TOPN) [16] is proposed on the base of HOONet [11]. It supports temporal knowledge description and object-oriented concepts. Modeling features in TOPN support describing and analyzing dynamic systems such as MAS and CMRS. Recently, some attempts have been conducted on modeling MAS by means of OPN [17].

Although Petri nets can be used to model and analyze different systems, they failed to model learning ability and the aging effects in dynamic systems. Recently, fuzzy timed Petri net (FTPN) [18] has been presented to solve these modeling problems. As a kind of reasoning and learning ability, fuzzy reasoning in FTPN can be considered as supporting autonomous judging or reasoning ability in MAS. In order to solve the reasoning ability and other modeling problems in large-scale MAS, fuzzy timed object-oriented Petri net (FTOPN) [19] is proposed on the base of TOPN [16] and FTPN [18]. In FTOPN, agent can be modeled as one FTOPN object with autonomy, situatedness and sociality. However, in FTOPN every agent should be modeled from common FTOPN objects and it needs generic FTOPN agent objects on the base of active objects.

This paper proposes a high level PN called fuzzy timed agent based Petri net (FTAPN) on the base of FTOPN [19]. As one of the typical active objects, ACTALK object is modeled by FTOPN and is introduced into FTAPN, which is used as generic agent object in FTAPN. The aim of FTAPN is to solve the agent or CMRS modeling ability problem and construct a bridge between MAS models and their implementations.

This paper is organized as the following. Section 2 reviews the relative preliminary notations quickly and Section 3 extends FTOPN to FTAPN on the base of ACTALK model. Section 4 discusses the learning which is important for representing dynamic behaviors in CMRS. Section 5 uses FTAPN to model one CMRS in the wafer etching procedure of circuit industry and makes some modeling analysis to demonstrate its benefits in modeling MAS. Finally, the conclusion and future work can be found in Section 6.

\section{Preliminary Notations}

In this section, the basic concepts of ACTALK are firstly reviewed, which is the relative concept in object-oriented concurrent programming. Then, the definitions of TOPN and FTOPN are introduced quickly, which are the basis of the research work in this paper.

\subsection{ACTALK}

\subsubsection{Active Objects [3]}

Object-oriented concurrent programming (OOCP) is one of the most appropriate and promising technologies for implementing or realizing agent based systems or MAS. Combining the agent concept and the object-oriented paradigm leads to the notion of agent-oriented programming [20]. The uniformity of objects' communication mechanisms provides facilities for implementing agent communication, and the concept of encapsulating objects or encapsulation support combining various agent granularities. Furthermore, the inheritance mechanism enables knowledge specialization and factorization.

The concept of an active object has been presented, which make it possible to integrate an object and activity (namely a thread or process). It also provides some degree of autonomy for objects in that it does not rely on external resources for activation. Thus, it provides a good basis for implementing agent based systems or MAS. However, similar to common objects in object-oriented systems, an active object's behavior still remains procedural and only reacts to message requests. More generally, the main feature of agent-based systems or MAS is autonomous. Agents should be able to complete tasks autonomously. That's to say, agents must be able to perform numerous functions or activities without external intervention over extended time periods. In order to achieve autonomy, adding to an active object a function that controls message reception and processing by considering its internal state is one of the effective realization methods $[21,22]$.

On one hand, for modelling and realizing MAS, there are two basic questions regarding how to build a bridge between implementing and modelling MAS requirements $[23,24]$. On the other hand, the facilities and techniques OOCP provides [25]:

I How can a generic structure define an autonomous agent's main features?

I How do we accommodate the highly structured OOCP model in this generic structure?

The active-object (or actor) concept has been introduced to describe a set of entities that cooperate and communicate through message passing. This concept brings the benefits of object orientation (for example, modularity and encapsulation) to distributed environments and provides object-oriented languages with some of the characteristics of open systems [26]. Based on these characteristics, various active object models have been proposed [27], and to facilitate implementing active-object systems, several frameworks have been pro- 
posed. ACTALK is one example.

When ACTALK is used to model and realize the MAS, there still exist the following shortcomings:

I Active object is not an autonomous agent. It only manifests the procedural actions.

I Although active object can communicate, they do not own the ability to reduce the decision to communicate or order other active objects.

I If one active object has not received the information from other active objects. It is still in none-active state.

In order to overcome the shortcomings mentioned above, the concept of active object has been proposed and a general agent framework [22,28]. A universal agent architecture has also been proposed so as to fulfil the modelling requirements of MAS [25], which can be used to model and analyze MAS deeply. For either agent-based systems or MAS, the method mostly is on the base of active objects. So in this chapter, the concept of active object is firstly reviewed quickly.

\subsubsection{ACTALK [29]}

One of the typical active objects is ACTALK. ACTALK is a framework for implementing and computing various active-object models into a single programming environment based on Smalltalk, which is an object-oriented programming language. ACTALK implements asynchronism, a basic principle of active-object languages, by queuing the received messages into a mailbox, thus dissociating message reception from interpretation. In ACTALK, an active object is composed of three component classes (see Figure 1), which are instances of the classes.

I Address encapsulates the active object's mailbox. It defines how to receive and queue messages for later interpretation.

I Activity represents the active object's internal activity and provides autonomy to the actor. It has a Smalltalk process and continuously removes messages from the mailbox, and the behavior component interprets the messages.

I ActiveObject represents the active object's behavior-that is, how individual messages are interpreted.

To build an active object with ACTALK, the algorithm must describe its behavior as a standard Smalltalk (OOCP) object. The active object using that behavior is created by sending the message active to the behavior:

active

"Creates an active object with self as corresponding behavior" Class

The activityClass and addressClass methods represent the default component classes for creating the activity and address components (along the factory method design pattern). To configure the framework of ACTALK means to define the components of its sub-classes. That's

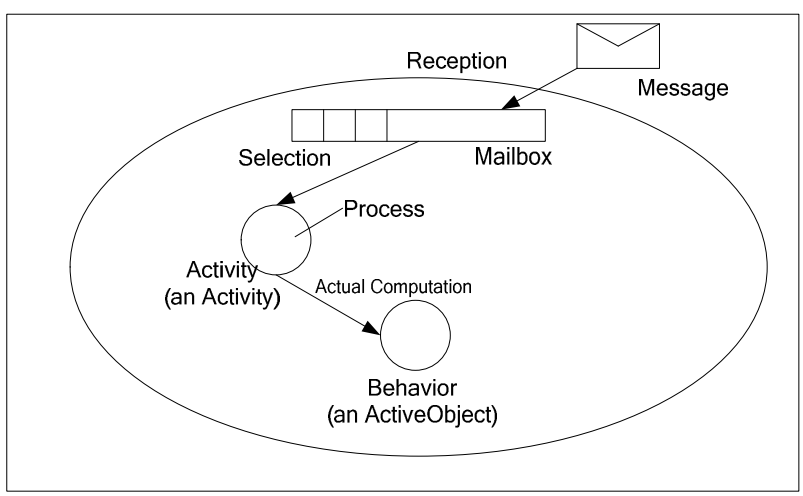

Figure 1. Components of an ACTALK active object.

to say, it allows users to define special active object models. So ACTALK is the basis to model agent based systems or MAS.

\subsection{Timed Object-Oriented Petri Net (TOPN)}

Formally TOPN is a four-tuple (OIP, ION, DD, SI), where (OIP, ION, DD) is an ordinary object Petri net-"HOONet" [30] and SI associates a static (firing) temporal interval SI: $\{\mathrm{o}\} \rightarrow[\mathrm{a}, \mathrm{b}]$ with each object o, where $\mathrm{a}$ and $\mathrm{b}$ are rationals in the range $0 \leqslant \mathrm{a} \leqslant \mathrm{b} \leqslant+\infty$, with $b \neq+\infty$. The four parts in TOPN have different function roles. Object identification place (OIP) is a unique identifier of a class. Internal timed object net (ION) is a net to depict the behaviors (methods) of a class. Data dictionary (DD) declares the attributes of a class in TOPN. And static time interval function (SI) binds the temporal knowledge of a class in TOPN. There are two kinds of places in TOPN. They are common places (represented as circles with thin prim) and abstract places (represented as circles with bold prim). Abstract places are also associated with a static time interval. Because at this situation, abstract places represent not only firing conditions, but also the objects with their own behaviors. So, abstract places (TABP) in TOPN also need to be associated with time intervals. One problem to be emphasized is that the tokens in abstract places need to have two colors at least. Before the internal behaviors of an abstract place object are fired, the color of tokens in it is one color (represented as hollow token in this paper). However, after fired, the color becomes the other one

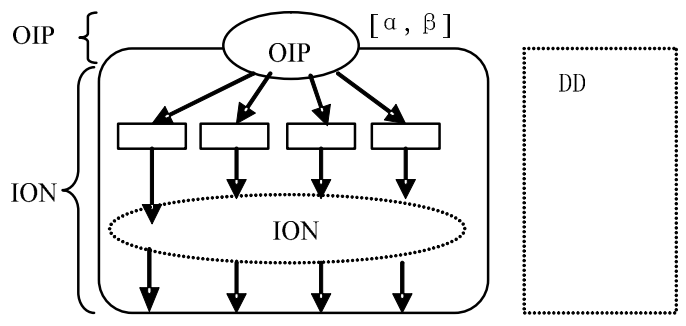

Figure 2. The general structure of TOPN. 


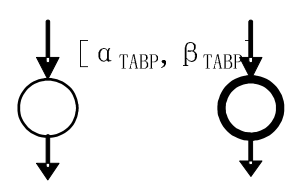

(a) Basic Place

(b) Abstract Place

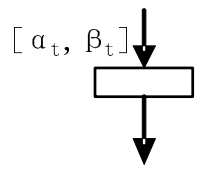

(c) Common Transition

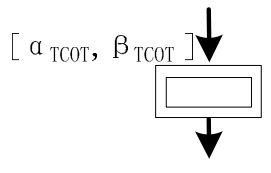

(d)

Communication Transition

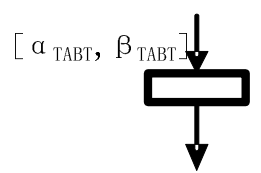

Figure 3. Places and transitions in TOPN.

(represented as liquid token in this paper). At this time, for the following transitions, it is just actually enabled. There are three kinds of transitions in TOPN. The timed primitive transition (represented as rectangles with thin prim) (TPIT), timed abstract transition (represented as rectangles with bold prim) (TABT) and timed communication transition (represented as rectangles with double thin prim) (TCOT).

Static time intervals change the behavior of TOPN just similar to a time Petri net in the following way. If an object o with $\mathrm{SI}(\mathrm{o})=[\mathrm{a}, \mathrm{b}]$ becomes enabled at time $\mathrm{I}_{0}$, then the object o must be fired in the time interval $\left[\mathrm{I}_{0}+\mathrm{a}\right.$, $\mathrm{I}_{0}+\mathrm{b}$ ], unless it becomes disabled by the removal of tokens from some input place in the meantime. The static earliest firing time of the object $o$ is a; the static latest firing time of $\mathrm{o}$ is $\mathrm{b}$; the dynamic earliest firing time (EFT) of $\mathrm{t}$ is $\mathrm{I}_{0}+\mathrm{a}$; the dynamic latest firing time (LFT) of $t$ is $\mathrm{I}_{0}+\mathrm{b}$; the dynamic firing interval of $\mathrm{t}$ is $\left[\mathrm{I}_{0}+\mathrm{a}, \mathrm{I}_{0}+\mathrm{b}\right]$.

The state of TOPN (Extended States- "ES") is a 3tuple, where ES = (M, I, path) consists of a marking M, a firing interval vector $I$ and an execution path. According to the initial marking $\mathrm{M}_{0}$ and the firing rules mentioned above, the following marking at any time can be calculated. The vector- "I" is composed of the temporal intervals of enabled transitions and TABPs, which are to be fired in the following states. The dimension of I equals to the number of enabled transitions and TABPs at the current state. The firing interval of every enabled transition or TABP can be got according to the calculation formula of EFT and LFT in TOPN [16].

For enabling rules in TOPN, two different situations exist. A transition $t$ in TOPN is said to be enabled at the current state (M, I, path), if each input place $\mathrm{p}$ of $\mathrm{t}$ contains at least the number of solid tokens equal to the weight of the directed arcs connecting $p$ to $t$ in the marking M. If the TABP object is marked with a hollow token, it is enabled. At this time, its ION is enabled. After the ION has been fired, the tokens in TABP are changed into solid ones.

An object o is said to be fireable in state (M, I, path) if it is enabled, and if it is legal to fire o next. This will be true if and only if the EFT of o is less than or equal to the LFT of all other enabled transitions. Of course, even with strong time semantics, o's being fireable in state (M, I, path) does not necessarily mean that $t$ will fire in the time interval I.

\subsection{Fuzzy Timed Object-Oriented Petri Net (FTOPN)}

Similar to FTPN [19], fuzzy set concepts are introduced into TOPN [16]. Then FTOPN is proposed, which can describe fuzzy timing effect in dynamic systems.

Definition 1: FTOPN is a six-tuple, FTOPN = $(\mathrm{OIP}$, ION, DD, SI, R, I) where

1) Suppose OIP = (oip, pid, $M_{0}$, status), where oip, pid, $\mathrm{M}_{0}$ and status are the same as those in HOONet [30] and TOPN [16].

I oip is a variable for the unique name of a FTOPN.

I pid is a unique process identifier to distinguish multiple instances of a class, which contains return address.

। $\mathrm{M}_{0}$ is the function that gives initial token distributions of this specific value to OIP.

I status is a flag variable to specify the state of OIP.

2) ION is the internal net structure of FTOPN to be defined in the following. It is a variant $\mathrm{CPN}$ that describes the changes in the values of attributes and the behaviors of methods in FTOPN.

3) DD formally defines the variables, token types and functions (methods) just like those in HOONet [30] and TOPN [16].

4) SI is a static time interval binding function, SI: $\{\mathrm{OIP}\} \rightarrow \mathrm{Q}^{*}$, where $\mathrm{Q}^{*}$ is a set of time intervals.

5) $\mathrm{R}:\{\mathrm{OIP}\} \rightarrow \mathrm{r}$, where $\mathrm{r}$ is a specific threshold.

6) I is a function of the time v. It evaluates the resulting degree of the abstract object firing.

Definition 2: An internal object net structure of TOPN, $\mathrm{ION}=\left(\mathrm{P}, \mathrm{T}, \mathrm{A}, \mathrm{K}, \mathrm{N}, \mathrm{G}, \mathrm{E}, \mathrm{F}, \mathrm{M}_{0}\right)$

1) $P$ and $T$ are finite sets of places and transitions with time restricting conditions attached respectively.

2) $\mathrm{A}$ is a finite set of arcs such that $\mathrm{P} \cap \mathrm{T}=\mathrm{P} \cap \mathrm{A}=$ $\mathrm{T} \cap \mathrm{A}=\Phi$.

3) $\mathrm{K}$ is a function mapping from $\mathrm{P}$ to a set of token types declared in DD.

4) N, G, and E mean the functions of nodes, guards, and arc expressions, respectively. The results of these functions are the additional condition to restrict the firing of transitions. So they are also called additional restricting conditions.

5) $\mathrm{F}$ is a special arc from any transitions to OIP, and notated as a body frame of ION.

6) $M_{0}$ is a function giving an initial marking to any place the same as those in HOONet [30] and TOPN [16].

Definition 3: A set of places in TOPN is defined as $P$ 
$=\mathrm{PIP} \cup \mathrm{TABP}$, where

1) Primary place PIP is a three-tuple: $P I P=(P, R, I)$, where

I $\mathrm{P}$ is the set of common places similar to those in PN [4,31].

2) Timed abstract place (TABP) is a six-tuple: TABP $=\mathrm{TABP}(\mathrm{pn}$, refine state, action, $\mathrm{SI}, \mathrm{R}, \mathrm{I})$, where

I $\mathrm{pn}$ is the identifier of the abstract timed place.

I refine state is a flag variable denoting whether this abstract place has been refined or not.

I action is the static reaction imitating the internal behavior of this abstract place.

I SI, R and I are the same as those in Definition 1.

Definition 4: A set of transitions in TOPN can be defined as $\mathrm{T}=\mathrm{TPIT} \cup \mathrm{TABT} \cup \mathrm{TCOT}$, where

1) Timed primitive transition TPIT = TPIT $(B A T, S I)$, where

I BAT is the set of common transitions.

2) Timed abstract transition TABT $=$ TABT (tn, refine state, action, SI), where

I tn is the name of this TABT.

3) Timed communication transition TCOT = TCOT (tn, target, comm type, action, SI).

I th is the name of TCOT.

I target is a flag variable denoting whether the behavior of this TCOT has been modeled or not. If target = "Yes", it has been modeled. Otherwise, if target = "No", it has not been modeled yet.

I comm type is a flag variable denoting the communication type. If comm type = "SYNC", then the communication transition is synchronous one. Otherwise, if comm type = "ASYN", it is an asynchronous communication transition.

4) SI is the same as that in Definition 1.

5) Refine state and action are the same as those in Definition 3 .

Similar to those in FTPN [19], the object $t$ fires if the foregoing objects come with a nonzero marking of the tokens; the level of firing is inherently continuous. The level of firing $(z(v))$ assuming values in the unit interval is governed by the following expression:

$$
z(v)=\left(\prod_{i=1}^{n}\left(r_{i} \rightarrow x_{i}\left(v^{\prime}\right)\right) s w_{i}\right) t \mathrm{I}(v)
$$

where $\mathrm{T}$ (or $\mathrm{t}$ ) denotes a t-norm while " $\mathrm{s}$ " stands for any s-norm. " $\mathrm{v}$ " is the time instant immediately following $\mathrm{v}$ '. More specifically, $\mathrm{x}_{\mathrm{i}}(\mathrm{v})$ denotes a level of marking of the $i^{\text {th }}$ place. The weight $w_{i}$ is used to quantify an input coming from the $i^{\text {th }}$ place. The threshold $r_{i}$ expresses an extent to which the corresponding place's marking contributes to the firing of the transition. The implication operator $(\rightarrow)$ expresses a requirement that a transition fires if the level of tokens exceeds a specific threshold (quantified here by $r_{i}$ ).
Once the transition has been fired, the input places involved in this firing modify their markings that is governed by the expression

$$
\mathrm{X}_{\mathrm{i}}(v)=\mathrm{X}_{\mathrm{i}}\left(v^{\prime}\right) \mathrm{t}(1-\mathrm{z}(v))
$$

(Note that the reduction in the level of marking depends upon the intensity of the firing of the corresponding transition, $\mathrm{z}(v)$.) Owing to the t-norm being used in the above expression, the marking of the input place gets lowered. The output place increases its level of tokens following the expression:

$$
\mathrm{y}(v)=\mathrm{y}\left(v^{\prime}\right) \mathrm{sz}(v)
$$

The s-norm is used to aggregate the level of firing of the transition with the actual level of tokens at this output place. This way of aggregation makes the marking of the output place increase.

The FTOPN model directly generalizes the Boolean case of TOPN and OPN. In other words, if $\mathrm{x}_{\mathrm{i}}(\mathrm{v})$ and $\mathrm{w}_{\mathrm{i}}$ assume values in $\{0,1\}$ then the rules governing the behavior of the net are the same as those encountered in TOPN.

\section{Agent Objects and Fuzzy Timed Agent Based Petri Nets}

The active object concept [29] has been proposed to describe a set of entities that cooperate and communicate through message passing. To facilitate implementing active object systems, several frameworks have been proposed. ACTALK is one of the typical examples. ACTALK is a framework for implementing and computing various active object models into one object-oriented language realization. ACTALK implements asynchronism, a basic principle of active object languages, by queuing the received messages into a mailbox, thus dissociating message reception from interpretation. In ACTALK,

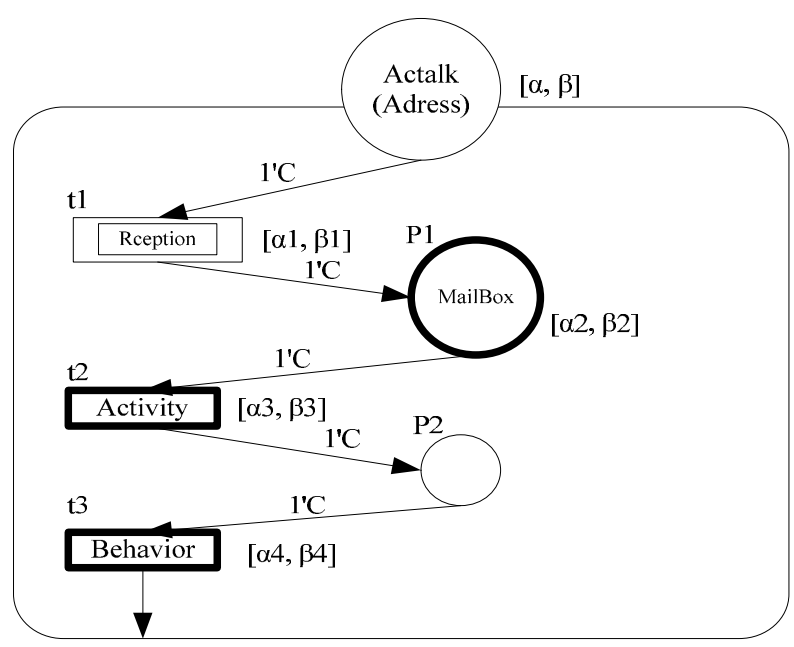

Figure 4. The FTOPN model of ACTALK. 
an active object is composed of three component classes: address, activity and activeObject [29].

ACTALK model is the basis of constructing active object models. However, active object model is the basis of constructing multi-agent system model or agent-based system model. So, as the modeling basis, ACTALK has been extended to different kinds of high-level agent models. Because of this, ACTALK is modeled in Fig.4 by FTOPN.

In Figure 4, OIP is the describer of the ACTALK model and also represents as the communication address. One communication transition is used to represent as the behavior of message reception. According to the communication requirements, it may be synchronous or asynchronous. If the message has been received, it will be stored in the corresponding mail box, which is one "first in and first out queue". If the message has been received, the next transition will be enabled immediately. So mail box is modeled as abstract place object in FTAPN. If there are messages in the mail box, the following transition will be enabled and fired. After the following responding activity completes, some active behavior will be conducted according to the message.

Figure 4 has described the ACTALK model based on FTOPN on the macroscopical level. The detailed definition or realization of the object "Activity" and "Behavior" can be defined by FTOPN in its parent objects in the lower level. The FTOPN model of ACTALK can be used as the basic agent object to model agent based systems. That is to say, if the agent based model-ACTALK model is used in the usual FTOPN modeling procedure, FTOPN has been extended to agent based modeling methodology. So it is called fuzzy timed agent based Petri net (FTAPN).

\section{Learning in Fuzzy Timed Agent Based Petri Nets}

The parameters of FTAPN are always given beforehand. In general, however, these parameters may not be available and need to be estimated just like those in FTPN [19]. The estimation is conducted on the base of some experimental data concerning marking of input and output places. The marking of the places is provided as a discrete time series. More specifically we consider that the marking of the output place(s) is treated as a collection of target values to be followed during the training process. As a matter of fact, the learning is carried out in a supervised mode returning to these target data.

The connections of the FTOPN (namely weights $w_{i}$ and thresholds $r_{i}$ ) as well as the time decay factors $\alpha_{i}$ are optimized (or trained) so that a given performance index $Q$ becomes minimized. The training data set consists of (a) initial marking of the input places $x_{i}(0), \ldots, x_{n}(0)$ and (b) target values - markings of the output place that are given in a sequence of discrete time moments, that is target $(0)$, target $(1), \ldots$, target $(\mathrm{K})$.

In FTAPN, the performance index $\mathrm{Q}$ under discussion assumes the form of Equation (4)

$$
Q=\sum_{k=1}^{K}(t \arg e t(k)-y(k))^{2}
$$

where the summation is taken over all time instants $(\mathrm{k}=1$, $2, \ldots, \mathrm{K})$.

The crux of the training in FTOPN models follows the general update formula in Equation (5) being applied to the parameters:

$$
\operatorname{param}\left(\text { iter+1) }=\operatorname{param}(\text { iter })-\gamma \nabla_{\text {param }} \boldsymbol{Q}\right.
$$

where $\gamma$ is a learning rate and $\nabla_{\text {param }} \boldsymbol{Q}$ denotes a gradient of the performance index taken with respect to all parameters of the net (here we use a notation param to embrace all parameters in FTOPN to be trained).

In the training of FTOPN models, marking of the input places is updated according to Equation (6):

$$
x_{i}{ }^{2}=x_{i}(0) T_{i}(k)
$$

where $\mathrm{T}_{\mathrm{i}}(\mathrm{k})$ is the temporal decay. And $\mathrm{T}_{\mathrm{i}}(\mathrm{k})$ complies with the form in Equation (7). In what follows, the temporal decay is modeled by an exponential function,

$$
T_{i}(k)=\left\{\begin{array}{c}
\exp \left(-\alpha_{i}\left(k-k_{i}\right)\right) \quad \text { if } \quad k>k_{i}, \\
0 \text { others }
\end{array}\right.
$$

The level of firing of the place can be computed as Equation (8):

$$
z=\left(\prod_{i=1}^{n} \quad\left(\left(r_{i} \rightarrow x_{i}^{\sim}\right) s w_{i}\right)\right)
$$

The successive level of tokens at the output place and input places can be calculated as that in Equation (9):

$$
\mathrm{y}(\mathrm{k})=\mathrm{y}(\mathrm{k}-1) s \mathrm{z}, \mathrm{x}_{\mathrm{i}}(\mathrm{k})=\mathrm{x}_{\mathrm{i}}(\mathrm{k}-1) t(1-\mathrm{z})
$$

We assume that the initial marking of the output place $\mathrm{y}(0)$ is equal to zero, $\mathrm{y}(0)=0$. The derivatives of the weights $w_{i}$ are computed as the form in Equation (9):

$$
\begin{aligned}
& \frac{\partial}{\partial w_{i}}(t \arg e t(k)-y(k))^{2} \\
& =-2\left(t \arg e t(k)-y(k) \frac{\partial y(k)}{\partial w_{i}}\right)
\end{aligned}
$$

where $\mathrm{i}=1,2, \ldots, \mathrm{n}$. Note that $\mathrm{y}(\mathrm{k}+1)=\mathrm{y}(\mathrm{k}) \mathrm{sz}(\mathrm{k})$.

\section{A Modeling Example}

\subsection{A CMRS Model}

In many typical integrated circuit manufacturing equipments such as etching tools, PVD, PECVD etc al., usually there is an EFAM platform which is made up of three Brooks Marathon Express (MX) [32] robots to transfer wafers to be processed. Among these three robots, 


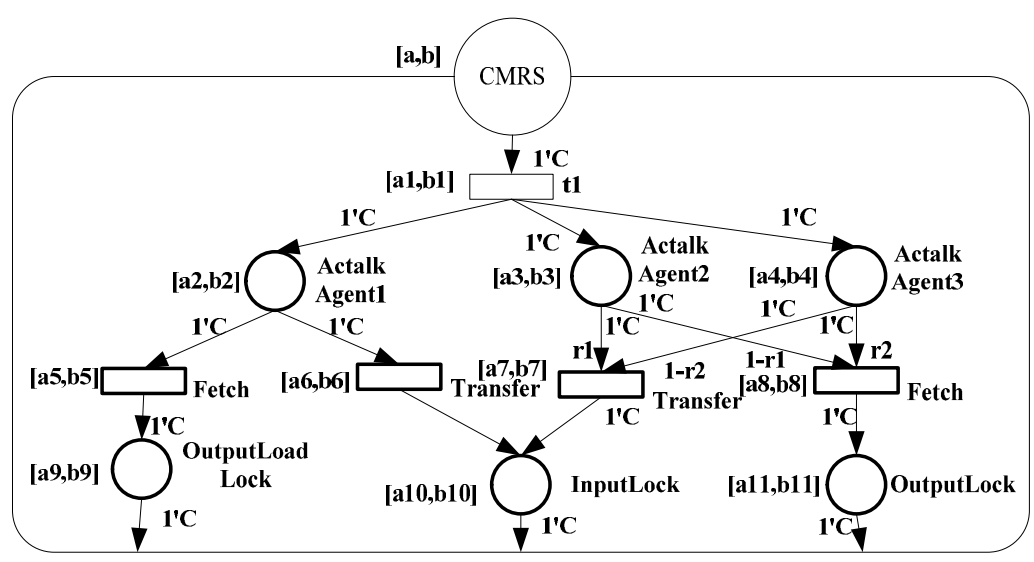

(a) The agent based FTAPN model

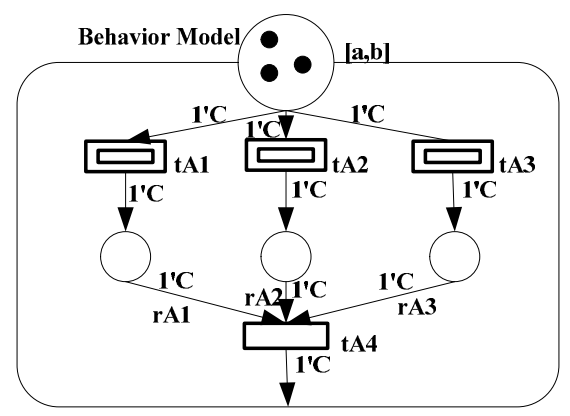

(b) The behavior model in every agent

Figure 5. The FTAPN model.

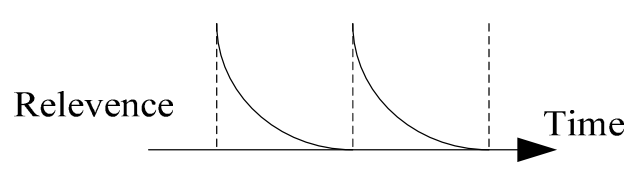

(a)

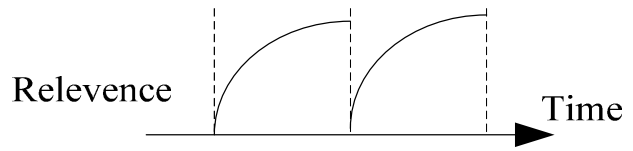

(b)

Figure 6. The relevance.

one is up to complete transferring wafers between atmospheric environment and vacuum environment, which is conducted in the atmospheric environment. In the vacuum environment, the other two robots are up to complete transferring one unprocessed wafer from the input lock to the chamber and fetch the processed wafer to the output lock in the vacuum environment. Any robot can be used to complete the transferring task at any time. If one robot is up to transfer one new wafer, the other will conduct the relative transferring or fetching task. They will not conflict with each other. Figure 5 depicts this CMRS FTAPN model, where three agent objects (ACTALK) are used to represent these three cooperative robots.

Figure 5(a) has depicted the whole FTAPN model. The agent object - "ACTALK" is used to represent every robot model. Different thresholds are used to represent the firing level of the behavior conducted by the corresponding robot (agent). They also satisfy the unitary requirements and change according to the fuzzy decision in the behavior of every agent in Figure 5(b). In the model of Figure 5(b), three communication transition objects are used to represent the behavior for getting different kinds of system states. These states include the state of the other robot, its own goal and its current state, which can be required by the conductions of the communication transitions tA1, tA2 and tA3. When one condition has been got, the following place will be marked. In order to make control decisions (transition object tA4) in time, all of these state parameters are required in the prescriptive time interval. However, the parameter arrival times complies with the rule in Figure 5(a). The other two kinds of information comply with that in Figure 5(b). After the decision, a new decision command with the conduction probability will be sent in this relative interval and it also affects which behavior (transfer or fetch) will be conducted by updating the threshold in Figure 5(a).

\subsection{Application Analysis}

Table 1 summarizes the main features of FTAPN and contrast these with the structures with which the proposed structures have a lot in common, namely MAS and FTOPN. It becomes apparent that FTAPN combines the advantages of both FTOPN in terms of their learning abilities and the glass-style of processing (and architectures) of MAS with the autonomy.

\section{Conclusions and Future Work}

CMRS is a kind of usual manufacturing equipments in manufacturing industries. In order to model, analyze and simulate this kind of systems, this paper proposes fuzzy timed agent based Petri net (FTAPN) on the base of FTOPN [19] and FTPN [18]. In FTAPN, one of the active objects-ACTALK is introduced and used as the basic agent object to model CMRS, which is a typical MAS. Every abstract object in FTOPN can be trained and reduced independently according to the modeling and analysis requirements for $\mathrm{OO}$ concepts supported in 
Table 1. MAS, FTOPN and FTAPN: A comparative analysis.

\begin{tabular}{llll}
\hline Characteristics & MAS & FTOPN & FTAPN \\
\hline & $\begin{array}{l}\text { Significant dynamic learn- } \\
\text { ing abilities. Dynamic learn- } \\
\text { ing and decision abilities are } \\
\text { supported in every autono- } \\
\text { mous agent. }\end{array}$ & $\begin{array}{l}\text { Significant learning abilities. } \\
\text { abilities are supported in dif- } \\
\text { ferent independent objects on }\end{array}$ & $\begin{array}{l}\text { Significant dynamic learning abilities. } \\
\text { sion abilities are supported in every } \\
\text { autonomous agent. }\end{array}$
\end{tabular}

Transparent knowledge representation (glass box processing style) the problem (its specification) is mapped directly onto the topology of the agent model. Additionally, agents deliver an essential feature of continuity required to cope with dynamic changes encountered in a vast array of problems (including autonomous decision tasks)
Glass Box Style (Transparent Knowledge Representation) and Black Box Processing is supported at the same time. The problem (its specification) is mapped directly onto the topology of FTOPN. Knowledge representation granularity reconfiguration reacts on the reduction of model size and complexity.
Glass Box Processing Style and Black Box Processing style are all supported. The problem (its specification) is mapped directly onto the topology of FTAPN, which can not only represent dynamic knowledge, but also deal with dynamic changes with well-defined semantics of agent objects, places, transitions, fuzzy and temporal knowledge.
FTOPN. The validity of this modeling method has been used to model Brooks CMRS platform in etching tools. The FTAPN can not only model complex MAS, but also be refined into the object-oriented implementation easily. It has provided a methodology to overcome the development problems in agent-oriented software engineering. At the same time, it can also be regarded as a conceptual and practical artificial intelligence (AI) tool for integrating MAS into the mainstream practice of software development.

State analysis needs to be studied in the future. An extended State Graph [16] has been proposed to analyze the state change of TOPN models. With the temporal fuzzy sets introduced into FTAPN, the certainty factor about object firing (state changing) needs to be considered in the state analysis.

\section{Acknowledgement}

This work is jointly supported by the National Nature Science Foundation of China (Grant No: 60405011, 60575057, 60875073) and National Key Technology R\&D Program of China (Grant No: 2009- BAG12A08).

\section{References}

[1] Y. U. Cao, A. S. Fukunaga, A. B. Kahng, and F. Meng, "Cooperative mobile robotics: Antecedents and directions," Autonomous Robots, Vol. 4, pp. 7-27, 1997.

[2] N. R. Jennings, K. Sycara, and M. Wooldridge, "A roadmap of agent research and development," Autonomous Agents and Multi-Agent Systems, Vol. 1, pp. 7-38, 1998.

[3] Z. Guessoum and J. P. Briot, "From active objects to autonomous agents," IEEE Concurrency, Vol. 7, No. 3, pp. 68-76, July-September 1999.

[4] T. Murata, "Petri nets and properties, analysis and applications," Proceedings of IEEE, Vol. 77, pp. 541-580, 1989.

[5] Y. L. Yao, "A Petri net model for temporal knowledge representation and reasoning," IEEE Transactions on Systems, Man and Cybernetics, Vol. 24, pp. 1374-1382, 1994.

[6] P. Merlin and D. Farber, "Recoverability of communication protocols-Implication of a theoretical study," IEEE Transactions on Communication, Vol. 24, pp. 1036-1043, 1976.

[7] J. Wang, Y. Deng, and M. Zhou, "Compositional time Petri nets and reduction rules," IEEE Transactions on Systems, Man and Cybernetics (Part B), Vol. 30, pp. 562-572, 2000.

[8] R. Bastide, "Approaches in unifying Petri nets and the object-oriented approach," Proceeding of the International Workshop on Object-Oriented Programming and Models of Concurrency, Turin, Italy, June, 1995, http:// eprints. kfupm.edu.sa/26256/.

[9] D. Harel and E. Gery, "Executable object modeling with statechart," Proceedings of the 18th International Conference on Software Engineering, Germany, pp. 246-257, March 1996.

[10] S. A. Schuman, "Formal object-oriented development," Springer, Berlin, 1997.

[11] J. E. Hong and D. H. Bae, "Software modeling and analysis using a hierarchical object-oriented Petri net," Information Sciences, Vol.130, pp. 133-164, 2000.

[12] E. Battiston, F. D. Cindio, and G. Mauri, "OBJSA nets: A class of high-level nets having objects as domains," APN'88, Lecture Notes in Computer Science, Vol. 340, pp. 20-43, 1988. 
[13] O. Biberstein and D. Buchs, "An object-oriented specification language based on hierarchical algebraic Petri nets," Proceedings of the IS-CORE Workshop Amsterdam, September 1994, http://citeseerx.ist.psu.edu/viewdoc/summary?doi=10.1.1.45.3092.

[14] C. Lakos and C. Keen, "LOOPN++: A new language for object-oriented Petri nets," Technical Report R94-4, Networking Research Group, University of Tasmania, Australia, April 1994.

[15] K. Jensen, "Coloured Petri nets: Basic concepts, analysis methods and practical use," Springer, Berlin, 1992.

[16] H. Xu and P. F. Jia, "Timed hierarchical object-oriented Petri net-part I: Basic concepts and reachability analysis," Lecture Notes in Artificial Intelligence (Proceedings of RSKT2006), Vol. 4062, pp. 727-734, 2006.

[17] W. Chainbi, "Multi-agent systems: A Petri net with objects based approach," Proceedings of IEEE/WIC/ACM International Conference on Intelligent Agent Technology, Beijing, pp. 429-432, 2004.

[18] W. Pedrycz and H. Camargo, "Fuzzy timed Petri nets, fuzzy sets and systems," Vol. 140, pp. 301-330, 2003.

[19] X. Hua and J. Peifa, "Fuzzy timed object-oriented Petri net," Artificial Intelligence Applications and Innovations II-Proceedings of AIAI2005, Springer, pp. 155-166, September 2005.

[20] Y. Shoham, "Agent-oriented programming," Artificial Intelligence, Vol. 60, No.1, pp. 139-159, 1993.

[21] J. P. Briot, "An experiment in classification and specialization of synchronization schemes, "Lecture Notes in Computer Science, No. 1107, pp. 227-249, 1996.

[22] T. Maruichi, M. Ichikawa, and M. Tokoro, "Decentralized AI," Modeling Autonomous Agents and Their Groups, Elsevier Science, Amsterdam, pp. 215-134, 1990.
[23] C. Castelfranchi, "A point missed in multi-agent, DAI and HCI," Lecture Notes in Artificial Intelligence, No. 890, pp. 49-62. 1995.

[24] L. Gasser, "An overview of DAI, " Distributed Artificial Intelligence, N. A. Avouris and L. Gasser, eds., Kluwer Academic, Boston, pp. 1-25, 1992.

[25] L. Gasser and J. P. Briot, "Object-oriented concurrent programming and distributed artificial intelligence," Distributed Artificial Intelligence, N. A. Avouris and L. Gasser, eds., Kluwer Academic, Boston, pp. 81-108, 1992.

[26] G. Agha and C. Hewitt, "Concurrent programming using actors: Exploiting large scale parallelism," Lecture Notes in Computer Science, S. N. Maheshwari, ed., SpringerVerlag, New York, No. 206, pp. 19-41, 1985.

[27] A. Yonezawa and M. Tokoro, "Object-oriented concurrent programming," The MIT Press, Cambrige, Mass., 1987.

[28] Y. Shoham, “Agent-oriented programming," Artificial Intelligence, Vol. 60, No.1, pp. 139-159, 1993.

[29] Z. Guessoum and J. P. Briot, "From active objects to autonomous agents," IEEE Concurrency, Vol. 7, No. 3, pp. 67-76, 1999.

[30] J. E. Hong and D. H. Bae, "Software modelling and analysis using a hierarchical object-oriented Petri net," Information Sciences, Vol. 130, pp. 133-164, 2000.

[31] J. L. Peterson, "Petri net theory and the modeling of systems," Prentice-Hall, N.Y., USA, 1991.

[32] J. H. Lee and T. E. Lee, "SECAM: A supervisory equipment control application model for integrated semiconductor manufacturing equipment, " IEEE Robotics \& Automation Magazine, Vol. 11, No. 1, pp. 41 - 58, 2004 .. 